of having its effect on my retinal circulation examined by one of my ophthal'mic colleagues, and then made my hospital visit without the consciousness of anything unusual in my mental condition, or any remarkable degree of somnolence perceptible, so far as I was aware, to my internes. Yet such a dose in the evening would have given me a quiet night if undisturbed, and $I$ have no doubt, if I could have yielded to it, would have done the same thing in the daytime. Opium, with its alkaloid, morphin, the first of narcotics, notwithstanding its undoubted power, its traditional vis dormitiva and the fact that sleep usually follows a sufficient dose after a varying interval, is not a desirable hypnotic in the strict sense of the word. In small doses it is untrustworthy, in many persons having the reverse of the desired effect, while in large ones its disadvantages far outweigh its benefits. Where, however, the want of sleep is dependent upon pain, the case is entirely different. It would be peculiarly dangerous in a case of neurasthenia, and I can hardly think of circumstances which would justify its use in a case of pure insomnia. Cannabis indica is hardly a hypnotic at all alone, or at least takes a long time to produce real sleep, but often does well in combination with bromid. I have used it occasionally alone with good effect when the dreams were depressing or annoying with the result, or coincidence, of their changing their character for the better.

Chloral, I think, has gone largely out of use. troduced as an anesthetic, then freely used in quite large doses as a hypnotic, it has been to a great extent abandoned for safer if less powerful drugs, and I judge from the catalogues of druge given me by patients that my opinion of its danger and uncertainty is shared by other physicians, though under the misnomer of "bromidia," which seems to obscure even in the professional mind the exact character of the preparation, it often appears upon the list of the narcotic "fiend." Of the immense number of other hypnotics of recent introduction, mostly of coal-tar or alcoholic origiı, each of which has in turn been brought forward as agreeable, certain and free from danger or disagreeable after-effects, I will only mention three.

I do not believe that the hypnotic is yet discovered or ever will be, which is at once trustworthy as producing the desired result and incapable of producing any unpleasant after-effects. Sulphonal comes as near to that standard as any drug with which I am acquainted, and used with care is little likely to do harm except that which attaches to any habit of the kind or dependence upon artificial aids to sleep. Nearly all, perbaps all, the cases, which, considering its widespread use, are not numerous, where dangerous conditions as evidenced either by hematoporphyrinuria or by long-continued subnarcotic effects have arisen, are the results either of doses singly too large or administered too close together, not allowing the blood to be free from it in the interval. The same remark might undoubtedly be made of other hypnotics. Its correctness may be shown by reference to the discussion at the Montreal meeting.

The tardiness and permanency of the action of sulphonal, undoubtedly a consequence of its difficult solubility and slow absorption, can not be looked upon as a disadvantage when properly allowed for. A second good night without a renewal of the dose, is by no means an exceptional occurrence even when the dose has not been excessive. It seldom fails in the dose of one gram if given well dissolved and early enough in the evening. In dose of thirty centigrams or less it is somewhat of a semiplacebo. The full dose should not be repeated on the same night, and it should not be given continuously, that is upon successive nights, for any length of time. If its use is to be prolonged beyond an emergency period of four or five days the interval should be more than forty-eight hours.

Trional is more soluble and rapid in its action, but seems to enjoy most of the advantages of sulphonal except the important one of having its effect last over two nights.

The peculiarities of each may be best utilized by reserving the sulphonal for a patient who has not slept for several nights and who, it is pretty clear, is not going to get a good night unassisted, so that the medicine may be given early, namely, at 6 or 7 P.M., in order that its effect may be developed at the regular time for going to sleep. Trional, on the other hand, may be kept for a case where sleep is likely to be well begun, but where it is likely to be broken off very early in the night and not renewed without a hypnotic. It is the preferable of the two for a provisional prescription, since the dose may be given at any time in the night necessary and the effect will not be too far distant. Paraldehyde is a rapidly acting and safe narcotic, probably coming much closer in its mode of action to alcohol than the coal-tar preparations. I have seen a case closely resembling a mild delirium tremens from the continued use of the drug in the hands of a patient not under professional care. The extremely disagreeable taste and smell may or may not be an advantage according to circumstances.

\section{THE CONTINUOUS USE OF DIGITALIN IN THE VASOMOTOR AND CARDIAC LESIONS OF SENILITY.}

Presented to the Section on Materia Medica, Pharmacy and Therapeutics at the Forty-ninth Annual Meeting of the American Medical Association, held at Denver, Colo., Jinne 7-10, 1898.

BY HENRY BEATES, JR., M.D.

PHILADELPHIA, PA.

Senility embraces that period of life during which one or more of the functions, as well as nutritional metamorphoses, when compared with the manifestations of the vital unit possessed by the individual, are recognized to be prematurely waning, and thus many are the instances of men and women, who, being endowed with vital energy sufficient to enroll them among the normal activities of advanced being, remain more or less disabled or invalided because of this premature failure on the part of some special organ or system to adequately discharge function. This retrograde process has an insidious origin, manifesting itself much earlier in life than we are prone to imagine, and is primarily operative, as a rule, in the vasomotor system. This great system has not, as yet, received the attentive study it should, and its direct relationship to functional activity, whether it. be of brain or special sense or organ, is such that it is not an exaggeration to state that life itself depends. thereon.

Active nutrition, then. in the fullest sense of the term, is that condition which determines the normal relationship of function to vital unit and is intimately associated with the perfect fulfillment of vasomotor function, because the distribution of pabulum to each cell, organ and system is directly dependent upun the. 
physiologic integrity of this nervo-sanguineous system. The first physiologic symptoms of senility will almost always be found with some portion of the vasomotor system, and that which is most objective consists in preternatural fullness of the superficial veins. This condition is indicative of far more advanced, although not visible, alteration of the minuter radicals, both of the arterioles and venules, and when present for some length of time, is associated with the symptoms of diminished and impaired function; therefore, we have slight defects in intellection; the details of everyday life are forgotten, and the victim finds it necessary to utilize the "memorandum tablet," instead of relying upon memory. Sleep is less refreshing and is interrupted by slight insomnia. Some failure in vision and obtunding of hearing co-exist, while respiration is habitually augmented in rate, and the ordinary activities of life, formerly free from any sense of discomfort, are associated with more or less dyspnea. The digestive functions are all less active than before, and the disturbances especially noticeable are those accompanying passive hyperemia and the consequent diminished absorption. The disturbances of hepatic functions manifest themselves in paroxysmal headache and pruritus; the itching of the nose is especially noticeable, and is often contemporaneously present with similar annoyances, located in areas of the back and lower extremities. Emaciation, while not marked, is pronounced, and the usual rotundity characteristic of the vigor of life, gives place to the well-known diminution in weight and wrinkling of the skin.

The function of the sphincters, and especially the vesical, is markedly impaired and the passive hyperemia of the kidney gives expression to its existence by albuminuria and the shedding of casts. The group of symptoms just enumerated, if the condition is well advanced, frequently assumes the clinical picture of organic disease, and thus has the writer seen what he erroneously believed to be senile softening of the brain, presenting typical symptoms, as demonstrated by a failure to recognize the members of family, ina bility to realize living at home, and re-living boyhood life, totally disappear under the treatment to be described; a fuct demonstrating that the failure of functional activity may be due, under these condi. tions, not to the death of the cell, but the absence of nutriment necessary to the maintenance of its vital phenomena. Of the cardiac conditions we have either fatty degeneration of the myocardium itself, cloudy swelling, or some type of retrograde change, either uncomplicated, which is exceedingly rare, or associated with gross lesions in the radicals and larger arteries. Indeed, the changes in the cardiac muscle are, in ninety-nine instances of the hundred, secondary to a primary lesion of the peripheral arterial system; this fact is worthy of especial emphasis and is to be considered in formulating a prognosis, other things being equal, of any given instance. Elsewhere will some facts associated with the physiology of the circulation be elaborated, and it will suffice in this connection to simply atate that, in senility, the propelling power of the arterial system being diminished, by reason of its vasomotor factors, but capable of restoration to the percentage longevity of the vital unit involved, will result in the establishment of lost equilibrium. The symptoms associated with what has hitherto been considered "senile heart" are, habitually augmented rate, diminution of resistance and edema of the extremities; of course, with these we have the bronchial disturbances, the falsely called "asthma," and, in advanced cases, various forms of dropsy. The treatment of these cases with digitalis, alcohol, strychnia, strophanthus and nitroglycerin finds its limit of usefulness far down the scale of possible achievement, and the premature death of all such, so afflicted, has been the outcome of secundum artem methods.

It is taught, that in the condition just described, arterial tension is increased and, therefore, digitalis should not be employed, because of producing rup. tures of the brittle and overfull arteries, and otherwise endangering the life of the sufferer. Careful study demonstrates that when the venous side of the circulation is tortuous and distended with blood, the arterial possesses less than its normal quantity, and while the aortic valves close with pronounced force, instead of being the result of arterial tension, this is actually dependent upon the weight of the column of blood, which not being propelled by what may be considered vermicular contraction, fills the non-elastic vessel as though it were an iron pipe; therefore, we have operative weight and not active force, and consequently diminished and not augmented arterial tension. What obtains in the aorta is an expression of the whole arterial vasomotor system and, consequently, we have this disturbed circulatory equilibrium, characterized by distended veins and relatively empty arteries. The cerebral phenomena so common in this condition manifest themselves in their worst type, by symptoms suggestive of apoplectic processes, and not uncommonly does the patient complain of localized numbness which, after a shorter or longer duration, culminates in confusion of thought, partial loss of consciousness, somnolency, confusion, loss of speech and transient paralysis of groups of muscles. This condition must not be confounded with true apoplexy, by which is meant rupture of a large arterial trunk, such as the middle meningeal or striate artery, because they are dependent upon either transient ischemia, that is, the terminal vessels fail to transmit blood to the part on account of the lack of propelling power, or to actual ruptures of venous radicals, directly due to the overdistention characteristic of the venous side.

A typical clinical illustration of an advanced case of senile cardio-vasomotor disease is that of an individual propped in a chair and completely disabled. For years nearly all of the symptoms briefly described have been gradually becoming more numerous and severe, until now he is distended with ansararca, the lower extremities ruptured and oozing serum freely, while the skin is red and glossy and the seat of ulceration. Respiration is imperfectly accomplished with constant effort, and is shallow and rapid, and the want of breath so marked that only a few words and disconnected sentences can be uttered at a time. The bronchial area is edematous and the lungs the seat of moist râles. Cough is persistent and exhausting, and sleep only indulged in for a few minutes at a time. The kidneys are almost passive, and only from two to four ounces of urine are secreted in the twenty-four hours. Exhaustion and distress are depicted on the countenance, but to the patient are not so keenly appreciable because of a partial obtund. ing of the brain. The cases from which this picture is drawn were the failures resulting from the secundum artem treatment, above mentioned, and although it is realized that the statement will be doubted, it is 
asserted that several who, years ago, were advanced to the stage depicted, are today free from all of these phenomena and able to be about as old, but comfortable seniles. The continuous use of "digitalin" in doses sufficiently large to restore lost circulatory equilibrium, is the treatment which brought about these truly wonderful results.

A few words about "digitalin": Unfortunately, this term is applied to a product in the shops which represents a complex mass or glucoside composed of one or more of the following active principles comtained in crude digitalis:

1 , digitalein, a glucoside occurring as a white amorphous bitter powder, soluble in water and alcohol; 2 , digitalin, a yellowish powder, soluble in alcohol, chloroform and 2000 parts of water; 3, digitalin German, really digitalein and an alleged inert crystallin derivative, known as digitin (this latter in a very small percentage); 4, digitonine; 5, digitalin kiliani, resolvable by treatment with acids into digitalose and digitaligenin; 6 , digitalin crystallin, soluble in alcohol and ether; 7, digitalin purified, which contains, also, digitalein, digitonin and digitalin kiliani; 8, digitonin; and 9, digitoxin.

When a prescription is ordered for "digitalin," the question may well be asked, what is supplied? Each of these derivatives possesses especial therapeutic power, which it is not the province of this contribution to consider; therefore, with simply indicating why there is such diversity of opinion entertained concerning the value of so-called digitalin, attention is directed to the fact that that derivative, the use of which this paper is the outcome, is a so-called digitalin, known as Digitalin German Merck. None other will effect the results set forth. Its dose is from one-tenth to one-half grain, from three to six times daily, as the severity of case requires.

If instances of advanced disease are encountered, and dissolution threatens when first seen, one-half a grain is safely exhibited hypodermically, and repeated in one hour; after that, two grains additional are administered by mouth, in quarter grain doses every two hours, should the emergency require. This bold plan has, in the writer's experience, several times averted impending death, and enabled restoration practicable, When the circulation has been restored by two or three days' treatment, the advantage is maintained by just sufficient of the remedy to secure permanent results. Skillfully used, this is maintained, as the title implies, continuously. A gentleman who, last October, was in the condition described and dying, has taken daily ever since, two grains a day. He now walks to my office, a distance of more than one mile, and returns to his home without any inconvenience whatever. After observing the effects of this treatment for years, it can be confidently asserted that no hypertrophy of the heart follows; also, that sclerosis of the arteries, not an expres. sion of calcareous infiltration or atheroma, but what may be viewed as an anchylosis, so to speak, frequently disappears.

This is due to the fact that the contraction and expansion essential to the calibrating function of the arteries being restored as a result of the action of "digitalin," normal functional activity and, consequently, nutrition of the coats is instituted; just as a stiffened articulation, by normal movements loses its rigidity, does an artery, similarly affected. The treatment must be practically continuous, because the senile changes having already become permanent are a constantly acting factor in the onward march. It must therefore be constantly held in abeyance and it can be, just as long a time as the life with which the vital unit of the individual was originally endowed.

RECOGNITION OF TEMPERAMENT: A FAC. TOR TO THE SELECTION OF REMEDIES AND THEIR DOSAGE IN DISEASE.

Presented to the Section on Materia Medica, Pharmacy and Therapeutics, at the Forty-ninth Annual Meeting of the American Medical Association, held at Denver, Colo., June 7-10, 1898.

\section{BY J. E. MOSES, M.D.}

KANSAS CTTY, MO.

Temperament may be defined as that quality, made manifest by the physical and mental characteristics of man. There are four distinct types of temperament: The nervous, the sanguine, the bilious, and the lymphatic. To these I will apply the term primary, because they are the base of all the combinations or compounds met with in the majority of people. A pure type of any one temperament is not often found, and when it is the characteristics are very marked. For the purpose of my paper it is necessary that I describe each temperament separately, and then take up the various compounds as they present themselves. Before taking up this description, I would state that I shall differ from all the authors that I have been able to consult, in that $I$ shall consider, not only the skin, the eyes, the hair, and the contour of the face as they do; but also the brain, the respiratory apparatus, the heart, liver, and digestive organs, as elements entering into the type or temperament under consideration.

Hippocrates and his followers claimed that the difference between men lay chiefly in the color of the eyes, hair and complexion, and that these could be sorted into four groups, in which all men might be placed. In the original idea or arrangement of temperament the mental qualities and traits of character were not considered, thereby losing sight of a very necessary factor in the recognition of an individual. A number of men have given this subject some consideration, and all have, in my humble opinion, fallen into the same error-overlooking the proportionate, relative sizes of the various organs of the body as elements of temperament; failing in this, they have been unable to present any system or plan that appealed in any measure to the medical profession as a possible aid in the treatment of disease. That these temperaments are easily recognized, when of a single type, is very true; but when in combinations of two or more, they become complex and difficult to master. As to a description of the temperaments, I know of no better than that formulated by Dr. Alex. Steward of Edinburgh, in his able work, entitled "Our Temperaments," and adding thereto certain ideas of $\mathrm{my}$ own which, I believe, materially influence the recognition of the characteristics of each.

THE NERVOUS TEMPERAMENT,

as the name would imply, is composed of a preponderance of nerve structure and mechanical velocity of action.

Physical characteristics. - Light brown hair; grey eyes: pale, clear complexion; face tapering from a high forehead and broad supra-orbital region to a narrow chin; the nose narrow; trunk slight and thin, never 